\title{
Defining and Managing High-Risk Multiple Myeloma: Current Concepts
}

\author{
Luciano J. Costa, MD, PhD, ${ }^{1}$ and Saad Z. Usmani, MD²
}

\section{ABSTRACT}

Multiple myeloma is a very heterogeneous disease. Despite advances in diagnostics and therapeutics, a subset of patients still experiences abbreviated responses to therapy, frequent relapses, and short survival and is considered to have high-risk multiple myeloma (HRMM). Stage III diagnosis according to the International Staging System; the presence of del(17p), $t(4 ; 14)$, or $t(14 ; 16)$ by fluorescence in situ hybridization; certain gene expression patterns; high serum lactic dehydrogenase level; and the presence of extramedullary disease at diagnosis are all considered indicators of HRMM. More recent evidence shows that patients who experience response to therapy but with a high burden of measurable residual disease or persistence of abnormal FDG uptake on PET/CT scan after initial therapy also have unfavorable outcomes, shaping the concept of dynamic risk assessment. Triplet therapy with proteasome inhibitors, immunomodulatory agents, and corticosteroids and autologous hematopoietic cell transplantation remain the pillars of HRMM therapy. Recent evidence indicates a benefit of immunotherapy with anti-CD38 monoclonal antibodies in HRMM. Future trials will inform the impact of novel immunotherapeutic approaches, including T-cell engagers, CAR T cells, and nonimmunotherapeutic approaches in HRMM. Those agents are likely to be deployed early in the disease course in the setting of riskand response-adapted trials.

J Natl Compr Canc Netw 2020;18(12):1730-1737 doi: $10.6004 /$ jnccn.2020.7673

${ }^{1}$ Division of Hematology and Oncology, Department of Medicine, O'Neal Comprehensive Cancer Center, University of Alabama at Birmingham, Birmingham, Alabama; and ${ }^{2}$ Plasma Cell Disorders Division, Department of Hematologic Oncology \& Blood Disorders, Levine Cancer Institute/Atrium Health, Charlotte, North Carolina.

\section{Defining High-Risk Multiple Myeloma}

Multiple myeloma (MM) is biologically heterogeneous, leading to wide variability in response to therapy and survival. The definitions of subsets of patients with high risk have evolved with diagnostic and therapeutic advances. This evolution has led to the first challenge in studying high-risk MM (HRMM): the multiplicity of imperfectly overlapping definitions.

\section{Traditional Staging}

As in most malignancies, the notion of risk in $\mathrm{MM}$ is intrinsically linked to traditional staging. The DurieSalmon Staging System (DSS), ${ }^{1}$ the first to be broadly used, was based on indicators of disease burden and renal function, but its performance was inadequate in the setting of more modern and effective therapies. A subsequent large multinational effort used robust statistical methodology applied to 10,750 patients to identify factors independently associated with survival in MM. The resulting International Staging System (ISS) identified serum levels of $\beta 2$-microglobulin and albumin as elements of a simple yet robust model to predict survival (Figure 1 ). ${ }^{2}$ Although the ISS outperformed the DSS, the rapid development of fluorescence in situ hybridization (FISH) in MM identified cytogenetic abnormalities as strong prognostic factors in MM, creating the need to revise the ISS in light of cytogenetic data and more modern therapeutics.

\section{Cytogenetics}

Conventional metaphase cytogenetics is often "normal" in MM, likely reflecting the low proliferation rate of plasma cells compared with normal marrow myeloid precursors. However, chromosome abnormalities are present in the most patients with MM. Common abnormalities not indicative of worse prognosis include trisomy of multiple odd-numbered chromosomes, $\mathrm{t}(11 ; 14)$ connecting the IgH promoter with the cyclin D1 gene, and del(13q). Abnormalities associated with worse prognosis include $\mathrm{t}(4 ; 14), \mathrm{t}(14 ; 16)$, and $\operatorname{del}(17 \mathrm{p})$ and together account for approximately $25 \%$ of patients with newly diagnosed MM (NDMM). An immense effort to refine $\mathrm{MM}$ staging with an 


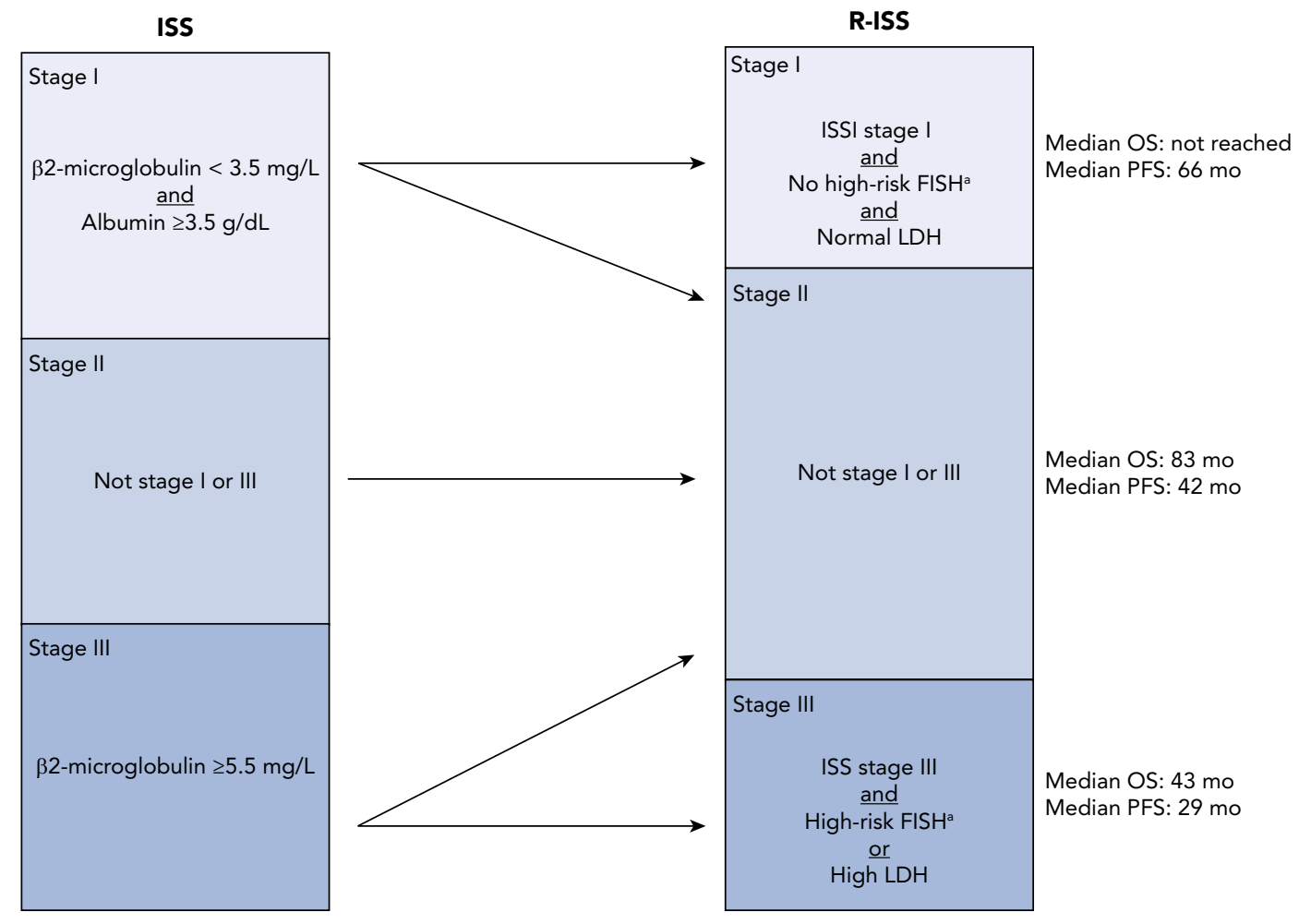

Figure 1. ISS and R-ISS for multiple myeloma.

Abbreviations: FISH, fluorescence in situ hybridization; ISS, International Staging System; LDH, lactate dehydrogenase; OS, overall survival; PFS, progression-free survival; R-ISS, Revised International Staging System.

aHigh-risk FISH abnormalities: $\mathrm{t}(4 ; 14), \mathrm{t}(14 ; 16)$, and/or del(17p).

assimilation of FISH information used data from 4,445 patients treated using immunomodulatory agents (IMiDs) and/or proteasome inhibitors (PIs) from 2005 to $2012 .^{3}$ The revised ISS (R-ISS) acknowledges the impact of $t(4 ; 14), t(14 ; 16)$, and del $(17 p)$ and the presence of elevated lactate dehydrogenase level on MM prognosis, defining a subset of approximately $15 \%$ of patients with R-ISS stage III disease and very poor prognosis (Figure 1).

Although the above classification of cytogenetic abnormalities in MM is solidified by the R-ISS, it is far from complete. Some less common abnormalities, particularly $\mathrm{t}(14 ; 20)$, present in approximately $1 \%$ to $2 \%$ of patients with MM, seem to indicate poor survival. ${ }^{4}$ The presence of gain (3 copies) or amplification ( $\geq 4$ copies) of chromosome 1q has been identified as being associated with worse prognosis than standard-risk MM in several datasets, and amplification of 1q21 is associated with bortezomib resistance. ${ }^{5,6}$ It is also now recognized that the presence of multiple high-risk genomic abnormalities (biallelic TP53 inactivation, gain/ amplification $C K S 1 B$ ) in association with ISS stage III disease ("double-hit" and "triple-hit") indicates a dismal prognosis. $^{7}$

\section{Gene Expression Profile}

The 1990s saw several advances in molecular biology, including the ability to use DNA microarrays to monitor the expression level of thousands of genes simultaneously. ${ }^{8}$ This technique, called gene expression profiling, was subsequently applied to CD138selected plasma cells from bone marrow aspirates by several groups in the 2000s for both disease biology characterization and prognostication. The Arkansas group defined 7 molecular subgroups of NDMM, ${ }^{9} 3$ of which were described as having the highest risk of early relapse: the MMSET gene overexpression, $M A F / M A F B$ overexpression, and proliferative gene overexpression subgroups. The Arkansas group also developed a prognostic model based on a 70-gene set that could identify an HRMM subset with an approximate median overall survival (OS) of 3 years. ${ }^{10}$ The HOVON/GMMG group generated a prognostic signature of 92 genes confirmed in sets of newly diagnosed and relapsed patients and were able to identify approximately $20 \%$ of patients with an OS of $<2$ years in both the transplant-eligible and nontransplant-eligible groups. ${ }^{11}$ The Arkansas high-risk signature is commercially available as MyPRS, and the 
HOVON signature is in commercial development as the SKY92 signature.

\section{Extramedullary Disease and Plasma Cell Leukemia}

Extramedullary manifestations of MM are an aggressive clinical phenotype characterized by malignant plasmal cells becoming independent of the bone marrow microenvironment. ${ }^{12,13}$ Clinical presentations include infiltration of other organs (lymph nodes, liver, lung, pleura, skin, central nervous system), known as extramedullary disease (EMD), or free circulation in the blood, referred to as plasma cell leukemia (PCL). When PCL presents at diagnosis, it is referred to as primary, whereas it is called secondary when presenting for the first time at disease relapse. ${ }^{14}$ PCL diagnosis is based on either percentage ( $\geq 20 \%$ of differential count) or absolute number $(\geq 2,000$ cells $/ \mu \mathrm{L})$. Interestingly, both $\operatorname{del}(17 \mathrm{p})$ and $\mathrm{t}(11$; 14) seem to be enriched in primary PCL. Both EMD and PCL are recognized as high-risk features at the time of diagnosis and at relapse. Median progression-free survival (PFS) for primary PCL has been reported to be 10 to 15 months but seems to be improving with more modern therapy, reaching 20 months in a recent series. ${ }^{15}$ Fiveyear survival in the literature is $<10 \%,{ }^{16}$ also improving with more modern therapy, autologous hematopoietic cell transplantation (AHCT), and maintenance. ${ }^{15}$ The incidence of EMD at diagnosis ranges from $1.7 \%$ to $4.5 \%$ and from $3.4 \%$ to $10 \%$ at relapse in different reported series. ${ }^{17,18}$ EMD must be distinguished from paramedullary extension from bone, because the latter is not considered an aggressive phenotype in the absence of other high-risk features (eg, FISH, elevated lactate dehydrogenase level).

\section{Dynamic Risk Assessment}

\section{Measurable Residual Disease and Posttherapy Imaging}

Given that some modern MM treatment regimens provide $>50 \%$ of complete response rates in NDMM, traditional response assessment becomes insufficient to describe short-term responses and to correlate them with long-term endpoints. ${ }^{19}$ Assessment of measurable residual disease (MRD) adds to traditional response criteria and is rapidly becoming an important, and often the primary, endpoint in trials for NDMM. Even when assessed by first-generation multiparameter flow cytometry with a sensitivity level of $10^{-4}$, the presence or absence of detectable MRD among patients with complete response clearly defines 2 groups with distinct PFS and OS (MRD-positive and MRD-negative, respectively), as shown in multiple studies and recent meta-analysis. ${ }^{20-22}$ The International Myeloma Working Group recognizes the sensitivity level of $10^{-5}$ assessed by next-generation flow ${ }^{23}$ or next-generation sequencing as the standard for MRD assessment and reporting. ${ }^{20} \mathrm{MRD}$ assessed at lower levels of sensitivity identifies subsets of patients with an even better prognosis. ${ }^{23,24}$ As opposed to most other HRMM definitions (ie, static and identifiable at presentation), MRD provides a tool for dynamic risk assessment, modifying the initial perception of risk for a certain group of patients. In fact, as shown in both the IFM $2009^{24}$ and PETHEMA/GEM2012MENOS65 ${ }^{23}$ clinical trials, patients with cytogenetic HRMM that becomes MRD-negative after initial therapy have favorable outcomes resembling those of patients with cytogenetic standard-risk MM who are MRD-negative. Conversely, among patients who remain MRD-positive, the initial risk category continues to predict outcomes. Although this finding does not support the pursuit of MRD negativity at all costs, it highlights how MRD after initial therapy may identify patients with high-risk cytogenetics and better prognosis.

Functional imaging after therapy provides additional prognostic information complementary to $\mathrm{MRD} .{ }^{25}$ As shown in the IFM 2009 trial, ${ }^{24}$ patients with either PET/ CT-positive disease or MRD levels $\geq 10^{-4}$ at the start of maintenance had inferior outcomes. Such a concept was confirmed more recently in the CASSIOPET study (a substudy of the CASSIOPEIA trial), in which PET/CT and MRD were compared before the start of maintenance therapy. ${ }^{25}$ Patients who were PET/CT-negative with MRD levels $<10^{-5}$ postconsolidation showed a trend toward longer PFS than those who were PET/CT-positive or had MRD levels $\geq 10^{-5}$ but without statistical significance, with a short follow-up time ( $\sim 19$ months median followup). ${ }^{25}$

Therefore, risk-based strategies deploying new therapies for patients with HRMM could focus on individuals who present with high-risk features (based on FISH, staging, or otherwise) and remain MRD-positive or PET/CT-positive after initial presentation.

\section{Early Relapse as a Hallmark of High Risk}

In addition to the high-risk features highlighted earlier, there is an increasing recognition that patients can experience early relapses despite not having HRMM features at diagnosis. This occurrence may represent disease evolution, either de novo or under duress of therapy, or simply represent the inadequate representation of disease from the single sample obtained at the time of diagnosis. The Arkansas group has shown that a pelvic bone biopsy may show standard-risk disease, but if CTguided biopsy is applied to other bony or EMD sites, then high-risk cytogenetic and genomic features can be observed. ${ }^{26}$ The consensus in the field is that early relapsed MM indicates poor outcomes. Patients experiencing relapse within 18 months of diagnosis are considered to have functional HRMM. 


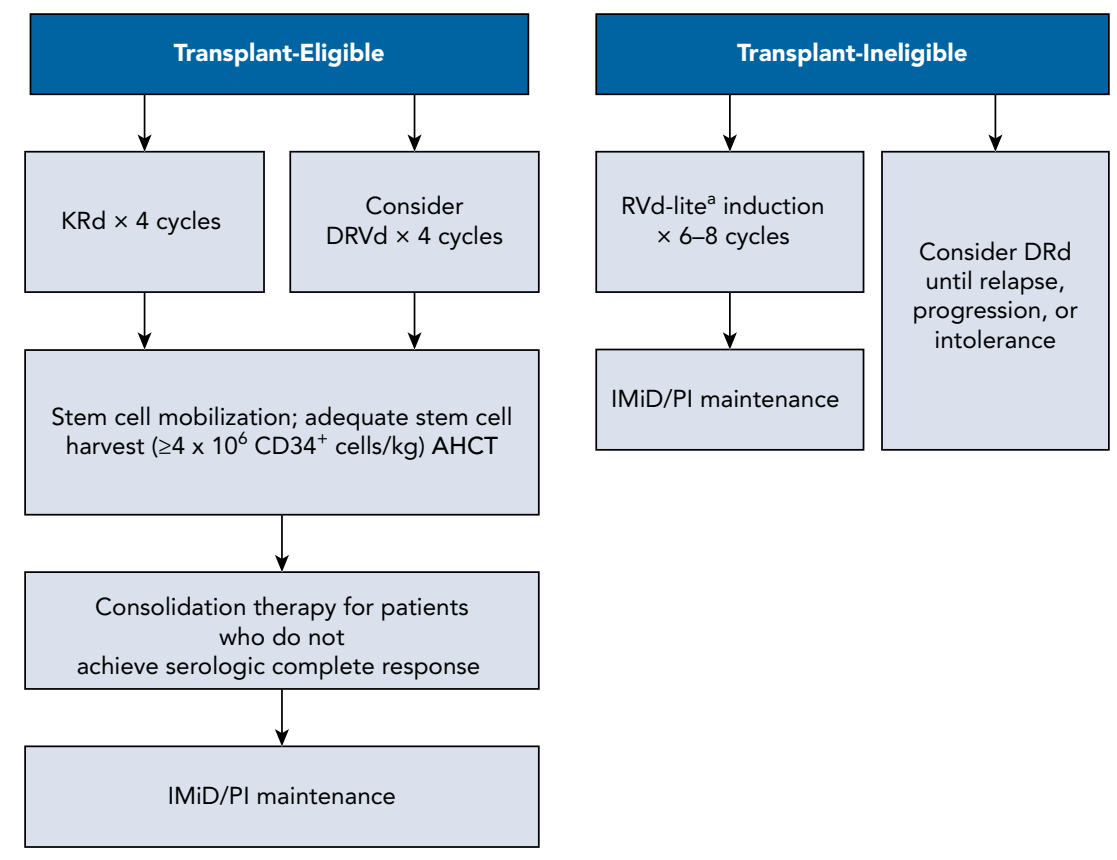

Figure 2. Suggested algorithm for initial management of patients with high-risk multiple myeloma.

Abbreviations: AHCT, autologous hematopoietic cell transplantation; d, dexamethasone; D, daratumumab; IMiD, immunomodulatory agent; K, carfilzomib; PI, proteasome inhibitor; $R$, lenalidomide; $V$, bortezomib.

aLenalidomide/Bortezomib/Dexamethasone using reduced doses of lenalidomide and dexamethasone and reduced frequency of subcutaneous bortezomib administration.

\section{Managing HRMM}

Even more challenging than identifying and classifying HRMM is developing adequate treatment strategies. Very few prospective trials have been performed exclusively in patients with HRMM. Moreover, this very heterogeneous population is underrepresented in nearly all clinical trials. Figure 2 contains a suggested algorithm for the initial treatment of HRMM.

\section{Pls and IMiDs in HRMM}

PIs, IMiDs, and corticosteroids are the pillars of modern MM therapy and for patients with HRMM..$^{27}$ The SWOG S0777 trial $^{28}$ comparing treatment with bortezomib/lenalidomide/ dexamethasone (VRd) versus lenalidomide/dexamethasone (Rd) in patients with NDMM showed improvement in PFS and OS for the VRd arm, including in the subset of patients with ISS stage III disease. For patients with high-risk FISH, median PFS was 38 months using VRd and 16 months using Rd, although this comparison was impaired by a low number of patients with high risk, which is a hindrance of most trials. Recently, the Emory group published their single-center experience of patients with NDMM who had received VRd as induction therapy, ${ }^{27}$ reporting a median PFS of 31.2 months and a median OS of 60.6 months for patients with HRMM.

Carfilzomib is a second-generation epoxyketone that differs structurally and mechanistically from the boronate-based PI bortezomib. The FORTE trial ${ }^{29}$ randomized 474 transplant-eligible patients with NDMM to receive carfilzomib/cyclophosphamide/ dexamethasone (KCd) induction (4 cycles) followed by AHCT followed by KCd consolidation (4 cycles) or carfilzomib/lenalidomide/dexamethasone (KRd) induction followed by AHCT followed by KRd consolidation or KRd for 12 cycles with deferred AHCT. KRd outperformed KCd among patients with R-ISS stage III disease in depth of response, including MRD. Subsequently, it was shown that despite a similar rate of response, KRd $\rightarrow$ AHCT $\rightarrow$ KRd led to a reduced risk of early progression and an increased rate of sustained MRD $<10^{-5}$ after 1 year compared with the same treatment substituting AHCT with 4 cycles of KRd among patients with R-ISS stage II/III disease. The recent ENDURANCE trial ${ }^{30}$ compared KRd versus VRd treatment in patients with standard risk and those with $\mathrm{t}(4 ; 14)$ without intent of upfront AHCT, yielding no superiority for either arm. No randomized comparison exists between the 2 regimens in patients with high risk.

\section{Monoclonal Antibodies in HRMM}

Monoclonal antibodies targeting CD38 (daratumumab) and SLAMF7 (elotuzumab) have been examined in several clinical trials for NDMM. The ALCYONE, ${ }^{31}$ MAIA, ${ }^{32}$ and CASSIOPEIA $^{33}$ phase III trials have compared the addition of daratumumab to bortezomib/melphalan/prednisone 
(VMP), Rd, and bortezomib/thalidomide/dexamethasone regimens, respectively. Although these trials met their primary endpoints, they showed no benefit of adding daratumumab in the small group of patients with cytogenetically defined HRMM included in each of these studies (ALCYONE, 14\%; MAIA, 14\%; CASSIOPEIA, 15.5\%). A more recent meta-analysis suggests that the addition of daratumumab across these frontline trials does improve PFS for patients with protocol-defined HRMM when compared with those in the control arms, but not to the same degree as in patients with standard-risk MM. ${ }^{34}$ Results of the randomized phase II SWOG-1211 study, the first enrichmentdesign trial for HRMM, were recently reported. ${ }^{35}$ The study compared the addition of elotuzumab to VRd versus VRd alone as part of induction followed by dose-attenuated maintenance, showing no difference in median PFS (31 vs 34 months) or median OS (68 months vs not reached).

\section{Autologous HCT}

High-dose melphalan with AHCT remains the standard of care for suitable patients who are newly diagnosed. However, patients with HRMM do not get the same benefit as those with standard risk. A Center for International Blood and Marrow Transplant Research (CIBMTR) analysis ${ }^{36}$ examining outcomes for 715 patients identified as high risk (del[17p], t[4;14], t[14;16], hypodiploidy, or chromosome $1 \mathrm{p}$ and $1 \mathrm{q}$ abnormalities) between 2008 and 2012 showed similar post-AHCT responses, but the responses were not sustained. The 3 -year post-AHCT PFS (37\% vs $49 \%$ ) and OS ( $72 \%$ vs $85 \%$ ) were lower in patients with HRMM compared with those with standard-risk MM. This analysis was critiqued for suboptimal induction regimens. Two registry analyses have reported on the role of AHCT in primary PCL. A European Society for Blood and Marrow Transplantation registry study ${ }^{37}$ of 272 patients with primary PCL treated between 1980 and 2006 reported median PFS and OS of 14.3 and 25.7 months, respectively. Similarly, CIBMTR ${ }^{38}$ reported on patients with primary PCL treated between 1995 and 2006, showing 3-year PFS and OS rates of 34\% and $64 \%$, respectively. In addition, the CIBMTR analysis also reported a trend toward superior OS in patients who received tandem AHCT compared with single AHCT, with 3-year PFS and OS rates of $84 \%$ and $56 \%$, respectively. These analyses were also critiqued for being performed in the pre-novel therapy era and therefore may represent patients with chemosensitive disease who were able to undergo an AHCT intervention.

EMN02/HO95 was a randomized phase III trial that compared VMP as a consolidation treatment compared with either single or tandem AHCT in NDMM. ${ }^{39}$ At median follow-up of 60.3 months, AHCT showed superior PFS with VMP (56.7 vs 41.9 months), including in patients with R-ISS stage III disease and those with high-risk cytogenetics. The recently reported STaMINA (BMT CTN 0702) phase III trial ${ }^{40,41}$ reported no difference in patients with HRMM treated using a tandem AHCT approach. However, an unplanned, post hoc "as treated" analysis indicated superior 6-year PFS for patients with high risk treated using tandem AHCT versus single AHCT ( $44 \%$ vs $32 \%$ ), indicating that a tandem approach may need to be prospectively explored in patients in the highrisk population. ${ }^{41}$ The Mayo Clinic group has also shown that patients with HRMM who achieve MRD negativity post-AHCT have superior PFS and OS compared with those who do not, but they noted an exception for patients with $\operatorname{del}(17 \mathrm{p})$ and/or $\geq 2$ high-risk cytogenetic abnormalities, ${ }^{42}$ speaking to the utility of depth of response as a dynamic risk assessment tool. Post-AHCT maintenance therapy in patients with high risk is an area of investigation. Singleagent lenalidomide yields inadequate results, and a combination of agents, particularly with the incorporation of PI, seems to yield superior results in nonrandomized studies. ${ }^{27}$

\section{Allogeneic HCT}

AHCT prolongs PFS and OS in patients with NDMM, but nearly all patients experience disease relapse. Allogeneic transplantation carries the potential benefit of graft-versusmyeloma but at the risk of increased nonrelapse mortality driven by conditioning regimen toxicity and graft-versus-host disease. Although allogeneic transplantation can provide long-term disease control in HRMM and may be curative in a few patients, randomized trials comparing allogeneic transplantation with AHCT predate modern risk classification systems and do not properly inform its systematic use in HRMM. ${ }^{43}$ The emergence of more effective MM therapies has discouraged the use of allogeneic HCT in NDMM outside clinical trials, whereas it remains an option for younger patients (age $<60$ years) with no or minimal comorbidities with relapsed HRMM when a suitable donor is available. ${ }^{44}$

\section{CAR T-Cell Therapy in HRMM}

B-cell maturation antigen (BCMA) is a transmembrane protein in the tumor necrosis receptor superfamily involved in the regulation of B-cell proliferation and survival and maturation/differentiation into PCs, making it an attractive target for immunotherapy. ${ }^{45} \mathrm{CAR} T$ cells are autologous T cells collected via apheresis and engineered to express the receptor targeting the desired antigen. The T cells are infused in a single episode, and their ability to expand in vivo results in persistence and ongoing immunity. BCMA-targeting CAR $\mathrm{T}$ cells produce fast and deep responses in most patients with heavily pretreated MM, including those with high-risk cytogenetics $(27 \%-41 \%$ of patients in trials) and EMD (10\%-39\% of patients in trials), yet the duration of response seems to be limited to less than a year in 
A

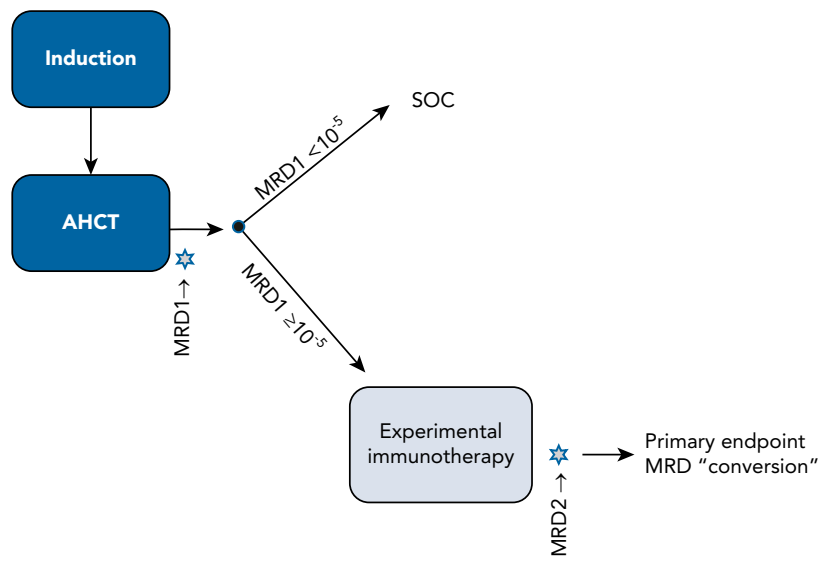

B

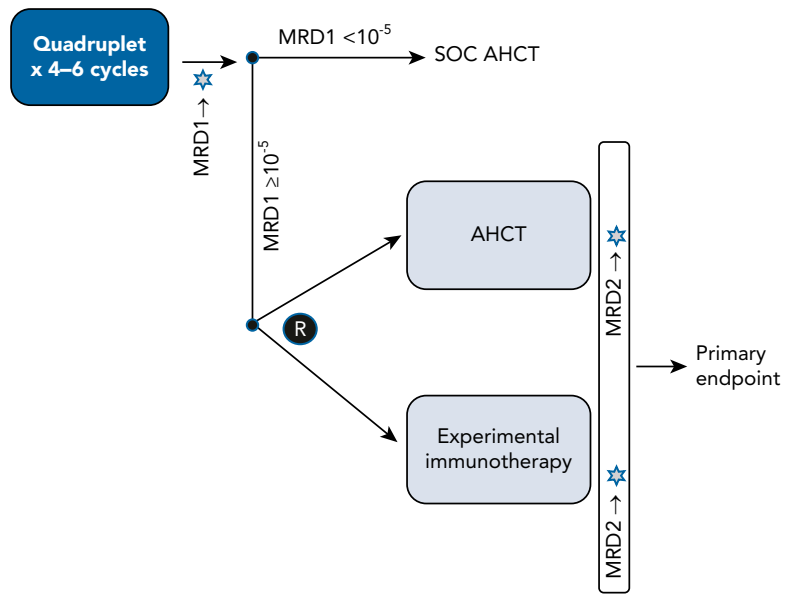

Figure 3. Possible strategies for early deployment of immunotherapy to address dynamic high-risk multiple myeloma. (A) Schema of a hypothetical trial using MRD to identify dynamic high-risk patients as candidates for experimental consolidation immunotherapy with the goal of MRD conversion. (B) Hypothetical clinical trial identifying dynamic high-risk patients and studying the role of AHCT versus experimental consolidation immunotherapy in a randomized fashion. Star indicates time of MRD assessment.

Abbreviations: AHCT, autologous hematopoietic cell transplantation; MRD, measurable residual disease; R, randomization; SOC, standard of care.

most patients. Such therapy has a manufacturing time window, which may not be realistic for patients with aggressive disease. Additional challenges include short-term toxicities (eg, cytokine release syndrome and neurotoxicity) and long-term complications (eg, ongoing cytopenia, organ dysfunction, and hypogammaglobinemia). ${ }^{46-48}$ These complications require continued monitoring in a tertiary care facility despite the advantage of a single infusion. A few trials have explored the role of upfront use of BCMA CAR T cells in HRMM with or without AHCT (ClinicalTrials.gov identifiers: NCT03549442, NCT03601078, NCT04196491, NCT03548207). CAR T cells may be deployed post-AHCT, in a setting of low disease burden, potentially limiting toxicity and leveraging the cytoreductive effect of AHCT. Use of CAR T cells in an upfront setting offers hope that more prolonged and perhaps definitive responses may be reached in some patients when CAR $\mathrm{T}$ cells are deployed early in the course of the disease.

\section{Strategies Addressing Dynamic Risk Assessment}

Achievement of MRD $<10^{-5}$ may abrogate initial risk classification in NDMM. The population of patients who are MRD-positive may still be very heterogeneous in their risk of relapse according to MRD burden. ${ }^{23,24}$ It is therefore important that future studies comparing strategies for consolidation or maintenance use dynamic risk assessment that incorporates MRD.

A possible approach is to assign patients with high (eg, $>10^{-5}$ ) MRD levels after initial therapy to be managed with experimental agents, with the shortterm objective of eliminating MRD and the long-term objective of improving duration of response. This strategy is particularly appealing for immunotherapeutic approaches, such as using CAR T cells and bispecific T-cell engagers (TCEs), in which much of the toxicity, particularly cytokine release syndrome, is driven by the burden of disease and will likely be ameliorated when deployed in settings with a relatively low amount of disease (Figure 3).

\section{New Drugs and Promising Approaches}

Several small molecules and immunotherapies are in clinical development for relapsed/refractory MM that have potential roles in newly diagnosed HRMM. Among the small molecules, venetoclax (an oral Bcl-2 inhibitor) seems to be more efficacious for treating MM with $\mathrm{t}(11$; 14) and Bcl-2 overexpression, ${ }^{49}$ and therefore may have a role in helping patients with PCL with those abnormalities. Similarly, selinexor (oral exportin-1 inhibitor) may have a role in treating patients with monoallelic $\operatorname{del}(17 p)$ MM based on its mechanism of action (nuclear membrane transport blocking causing retention of p53, other tumor suppressor proteins, and oncogenic mRNAs in the nucleus ${ }^{50}$ ) and available evidence in clinical trials showing its efficacy in patients with relapsed HRMM. ${ }^{51-53}$

In addition to CAR T cells, BCMA has been the target for other immunotherapeutic approaches, particularly TCEs and antibody-drug conjugates (ADCs).$^{54}$ Currently available clinical data for these agents are dominated by trials in patients with disease refractory to IMiDs, PIs, and CD38 monoclonal antibodies, a population that can be considered high risk in its entirety. At the highest safe 
doses tested, the BCMA TCEs AMG420, ${ }^{55}$ teclistamab (ClinicalTrials.gov identifier: NCT04557098), and CC93269 (NCT03486067) all produced responses in more than twothirds of patients. ${ }^{54}$ Cytokine release syndrome and infections were the most noteworthy toxicities. The ADC belantamab mafodotin produced a response rate of $33 \%$, with a similar rate in patients with high-risk cytogenetics. The main toxicities were cytopenias and keratopathy. ${ }^{54}$

The next few years are likely to bring further refinement to the understanding of the biology of HRMM and the optimization of risk stratification, along with risk-specific clinical trials. Moreover, we expect to see data maturity; new applications of CAR T-cell therapy, TCEs, and ADCs; and the fast development of new immunotherapeutic and nonimmunotherapeutic targets. Together, these advances may bring breakthroughs in this difficult and heterogeneous group of patients with MM.

Submitted July 29, 2020; accepted for publication October 15, 2020

Disclosures: Dr. Costa has disclosed that he receives honoraria from Amgen, Bristol-Myers Squibb, AbbVie, and Janssen, and grant/research support from Amgen, Bristol-Myers Squibb, AbbVie, and Janssen. Dr. Usmani has disclosed that he receives honoraria from Celgene/Bristol-Myers Squibb, Amgen, Merck \& Co., Inc., GlaxoSmithKline, Seattle Genetics, Takeda, Sanofi, and Janssen, and grant/research support from Onyx, Janssen, Sanofi, Array BioPharma, Pharmacyclics, Takeda, Celgene, and Bristol-Myers Squibb.

Correspondence: Luciano J. Costa, MD, PhD, Division of Hematology and Oncology, Department of Medicine, University of Alabama at Birmingham, 1802 6th Avenue South, Birmingham, AL 35294. Email: ljcosta@uabmc.edu

\section{References}

1. Durie BG, Salmon SE. A clinical staging system for multiple myeloma. Correlation of measured myeloma cell mass with presenting clinical features, response to treatment, and survival. Cancer 1975;36:842-854.

2. Greipp PR, San Miguel J, Durie BG, et al. International staging system for multiple myeloma. J Clin Oncol 2005;23:3412-3420.

3. Palumbo A, Avet-Loiseau H, Oliva S, et al. Revised International Staging System for Multiple Myeloma: a report from International Myeloma Working Group. J Clin Oncol 2015;33:2863-2869.

4. Ross FM, Chiecchio L, Dagrada G, et al. The t $(14 ; 20)$ is a poor prognostic factor in myeloma but is associated with long-term stable disease in monoclonal gammopathies of undetermined significance. Haematologica 2010;95:1221-1225.

5. Shaughnessy JD Jr, Qu P, Usmani S, et al. Pharmacogenomics of bortezomib test-dosing identifies hyperexpression of proteasome genes, especially PSMD4, as novel high-risk feature in myeloma treated with Total Therapy 3. Blood 2011;118:3512-3524.

6. Giri S, Huntington SF, Wang R, et al. Chromosome 1 abnormalities and survival of patients with multiple myeloma in the era of novel agents. Blood Adv 2020;4:2245-2253.

7. Walker BA, Mavrommatis K, Wardell CP, et al. A high-risk, double-hit, group of newly diagnosed myeloma identified by genomic analysis. Leukemia 2019;33:159-170.

8. Schena M, Shalon D, Davis RW, et al. Quantitative monitoring of gene expression patterns with a complementary DNA microarray. Science 1995;270:467-470.

9. Zhan F, Huang Y, Colla S, et al. The molecular classification of multiple myeloma. Blood 2006;108:2020-2028.

10. Zhan F, Barlogie B, Mulligan G, et al. High-risk myeloma: a gene expression based risk-stratification model for newly diagnosed multiple myeloma treated with high-dose therapy is predictive of outcome in relapsed disease treated with single-agent bortezomib or high-dose dexamethasone. Blood 2008;111:968-969.

11. Kuiper R, Broyl A, de Knegt $Y$, et al. A gene expression signature for high-risk multiple myeloma. Leukemia 2012;26:2406-2413.

12. Bhutani M, Foureau DM, Atrash $\mathrm{S}$, et al. Extramedullary multiple myeloma. Leukemia 2020;34:1-20.

13. Rajkumar SV, Dimopoulos MA, Palumbo A, et al. International Myeloma Working Group updated criteria for the diagnosis of multiple myeloma. Lancet Oncol 2014;15:e538-548.

14. Fernández de Larrea C, Kyle RA, Durie BG, et al. Plasma cell leukemia: consensus statement on diagnostic requirements, response criteria and treatment recommendations by the International Myeloma Working Group. Leukemia 2013;27:780-791.

15. Mina R, Joseph NS, Kaufman JL, et al. Survival outcomes of patients with primary plasma cell leukemia (pPCL) treated with novel agents. Cancer 2019;125:416-423.

16. Gonsalves WI, Rajkumar SV, Go RS, et al. Trends in survival of patients with primary plasma cell leukemia: a population-based analysis. Blood 2014; 124:907-912.

17. Usmani SZ, Heuck C, Mitchell A, et al. Extramedullary disease portends poor prognosis in multiple myeloma and is over-represented in high-risk disease even in the era of novel agents. Haematologica 2012;97: 1761-1767.

18. Varettoni M, Corso A, Pica G, et al. Incidence, presenting features and outcome of extramedullary disease in multiple myeloma: a longitudinal study on 1003 consecutive patients. Ann Oncol 2010;21:325-330.

19. Lahuerta JJ, Paiva B, Vidriales MB, et al. Depth of response in multiple myeloma: a pooled analysis of three PETHEMA/GEM clinical trials. J Clin Oncol 2017;35:2900-2910.

20. Kumar S, Paiva B, Anderson KC, et al. International Myeloma Working Group consensus criteria for response and minimal residual disease assessment in multiple myeloma. Lancet Oncol 2016;17: e328-346.

21. Munshi NC, Avet-Loiseau H, Rawstron AC, et al. Association of minimal residual disease with superior survival outcomes in patients with multiple myeloma: a meta-analysis. JAMA Oncol 2017;3:28-35.

22. Martinez-Lopez J, Lahuerta JJ, Pepin F, et al. Prognostic value of deep sequencing method for minimal residual disease detection in multiple myeloma. Blood 2014;123:3073-3079.

23. Paiva B, Puig N, Cedena MT, et al. Measurable residual disease by nextgeneration flow cytometry in multiple myeloma. J Clin Oncol 2020;38: 784-792.

24. Perrot A, Lauwers-Cances V, Corre J, et al. Minimal residual disease negativity using deep sequencing is a major prognostic factor in multiple myeloma. Blood 2018;132:2456-2464.

25. Moreau P, Zweegman S, Perrot A, et al. Evaluation of the prognostic value of positron emission tomography-computed tomography (PET-CT) at diagnosis and follow-up in transplant-eligible newly diagnosed multiple myeloma (TE NDMM) patients treated in the phase 3 CASSIOPEIA study: results of the CASSIOPET companion study [abstract]. Blood 2019; 134(Suppl):Abstract 692

26. Rasche L, Chavan SS, Stephens OW, et al. Spatial genomic heterogeneity in multiple myeloma revealed by multi-region sequencing. Nat Commun 2017;8:268.

27. Joseph NS, Kaufman JL, Dhodapkar MV, et al. Long-term follow-up results of lenalidomide, bortezomib, and dexamethasone induction therapy and risk-adapted maintenance approach in newly diagnosed multiple myeloma. J Clin Oncol 2020;38:1928-1937.

28. Durie BGM, Hoering A, Abidi $M H$, et al. Bortezomib with lenalidomide and dexamethasone versus lenalidomide and dexamethasone alone in patients with newly diagnosed myeloma without intent for immediate autologous stem-cell transplant (SWOG S0777): a randomised, openlabel, phase 3 trial. Lancet 2017;389:519-527.

29. Gay F, Cerrato C, Petrucci MT, et al. Efficacy of carfilzomib lenalidomide dexamethasone (KRd) with or without transplantation in newly diagnosed myeloma according to risk status: results from the FORTE trial [abstract]. J Clin Oncol 2019;37(Suppl):Abstract 8002.

30. Kumar SK, Jacobus SJ, Cohen AD, et al. Carfilzomib or bortezomib in combination with lenalidomide and dexamethasone for patients with newly diagnosed multiple myeloma without intention for immediate autologous stem-cell transplantation (ENDURANCE): a multicentre, 
open-label, phase 3, randomised, controlled trial. Lancet Oncol 2020;21: 1317-1330.

31. Mateos MV, Cavo M, Blade J, et al. Overall survival with daratumumab, bortezomib, melphalan, and prednisone in newly diagnosed multiple myeloma (ALCYONE): a randomised, open-label, phase 3 trial. Lancet 2020;395:132-141.

32. Facon T, Kumar S, Plesner T, et al. Daratumumab plus lenalidomide and dexamethasone for untreated myeloma. N Engl J Med 2019;380: 2104-2115.

33. Moreau P, Attal M, Hulin C, et al. Bortezomib, thalidomide, and dexamethasone with or without daratumumab before and after autologous stem-cell transplantation for newly diagnosed multiple myeloma (CAS SIOPEIA): a randomised, open-label, phase 3 study. Lancet 2019;394: 29-38.

34. Giri S, Grimshaw A, Bal S, et al. Efficacy of daratumumab in the treatment of multiple myeloma with high-risk cytogenetics: meta-analysis of randomized phase III trials [abstract]. J Clin Oncol 2020;38(Suppl):Abstract 8540.

35. Usmani SZ, Ailawadhi $\mathrm{S}$, Sexton $\mathrm{R}$, et al. Primary analysis of the randomized phase II trial of bortezomib, lenalidomide, dexamethasone with/ without elotuzumab for newly diagnosed, high-risk multiple myeloma (SWOG-1211) [abstract]. J Clin Oncol 2020;38(Suppl):Abstract 8507.

36. Scott EC, Hari P, Sharma M, et al. Post-transplant outcomes in high-risk compared with non-high-risk multiple myeloma: a CIBMTR analysis. Biol Blood Marrow Transplant 2016;22:1893-1899.

37. Drake MB, lacobelli $S$, van Biezen $A$, et al. Primary plasma cell leukemia and autologous stem cell transplantation. Haematologica 2010;95:804-809.

38. Mahindra A, Kalaycio ME, Vela-Ojeda J, et al. Hematopoietic cell transplantation for primary plasma cell leukemia: results from the Center for International Blood and Marrow Transplant Research. Leukemia 2012; 26:1091-1097.

39. Cavo M, Gay F, Beksac M, et al. Autologous haematopoietic stem-cell transplantation versus bortezomib-melphalan-prednisone, with or without bortezomib-lenalidomide-dexamethasone consolidation therapy, and lenalidomide maintenance for newly diagnosed multiple myeloma (EMN02/HO95): a multicentre, randomised, open-label, phase 3 study. Lancet Haematol 2020;7:e456-468.

40. Stadtmauer EA, Pasquini MC, Blackwell B, et al. Autologous transplantation, consolidation, and maintenance therapy in multiple myeloma: results of the BMT CTN 0702 trial. J Clin Oncol 2019;37:589-597.

41. Hari P, Pasquini MC, Stadtmauer EA, et al. Long-term follow-up of BMT CTN 0702 (STaMINA) of postautologous hematopoietic cell transplantation (autoHCT) strategies in the upfront treatment of multiple myeloma (MM) [abstract]. J Clin Oncol 2020;38(Suppl):Abstract 8506.

42. Chakraborty R, Muchtar E, Kumar SK, et al. Impact of post-transplant response and minimal residual disease on survival in myeloma with highrisk cytogenetics. Biol Blood Marrow Transplant 2017:23:598-605.
43. Costa LJ, lacobelli S, Pasquini MC, et al. Long-term survival of $1338 \mathrm{MM}$ patients treated with tandem autologous vs. autologous-allogeneic transplantation. Bone Marrow Transplant 2020;55:1810-1816.

44. Greil C, Engelhardt M, Ihorst G, et al. Allogeneic transplantation of multiple myeloma patients may allow long-term survival in carefully selected patients with acceptable toxicity and preserved quality of life. Haematologica 2019;104:370-379.

45. Shah N, Chari A, Scott E, et al. B-cell maturation antigen (BCMA) in multiple myeloma: rationale for targeting and current therapeutic approaches. Leukemia 2020;34:985-1005.

46. Raje N, Berdeja J, Lin Y, et al. Anti-BCMA CAR T-cell therapy bb2121 in relapsed or refractory multiple myeloma. N Engl J Med 2019;380:1726-1737.

47. Madduri D, Usmani SZ, Jagannath $S$, et al. Results from CARTITUDE-1: phase $1 \mathrm{~b} / 2$ study of JNJ-4528, a CAR-T cell therapy directed against $\mathrm{B}$-cell maturation antigen (BCMA), in patients with relapsed and/or refractory multiple myeloma (R/R MM) [abstract]. Blood 2019;134(Suppl): Abstract 577.

48. Mailankody $\mathrm{S}$, Jakubowiak AJ, Htut M et al. Orvacabtagene autoleuce (orva-cel), a B-cell maturation antigen (BCMA)-directed CAR T cell therapy for patients (pts) with relapsed/refractory multiple myeloma (RRMM): update of the phase 1/2 EVOLVE study (NCT03430011) [abstract]. J Clin Oncol 2020;38(Suppl):Abstract 8504.

49. Kumar S, Kaufman JL, Gasparetto C, et al. Efficacy of venetoclax as targeted therapy for relapsed/refractory $\mathrm{t}(11 ; 14)$ multiple myeloma. Blood 2017;130:2401-2409

50. Tai YT, Landesman Y, Acharya C, et al. CRM1 inhibition induces tumor cell cytotoxicity and impairs osteoclastogenesis in multiple myeloma: molecular mechanisms and therapeutic implications. Leukemia 2014;28:155-165.

51. Chari A, Vogl DT, Gavriatopoulou M, et al. Oral selinexor-dexamethasone for triple-class refractory multiple myeloma. N Engl J Med 2019;381: 727-738.

52. Nooka AK, Yee AJ, Huff CA, et al. Influence of cytogenetics in patients with relapsed refractory multiple myeloma treated with oral selinexor and dexamethasone: a post-hoc analysis of the STORM study [abstract]. Blood 2019;134(Suppl):Abstract 1872.

53. Dimopoulos MA, Delimpasi S, Simonova M, et al. Weekly selinexor, bortezomib, and dexamethasone (SVd) versus twice weekly bortezomib and dexamethasone $(\mathrm{Vd})$ in patients with multiple myeloma (MM) after one to three prior therapies: initial results of the phase III BOSTON study [abstract]. J Clin Oncol 2020;38(Suppl):Abstract 8501

54. Lonial S, Lee HC, Badros A, et al. Belantamab mafodotin for relapsed or refractory multiple myeloma (DREAMM-2): a two-arm, randomised, openlabel, phase 2 study. Lancet Oncol 2020;21:207-221.

55. Topp MS, Duell J, Zugmaier G, et al. Anti-B-cell maturation antigen BiTE molecule AMG 420 indices responses in multiple myeloma. J Clin Onco 2020:38:775-783. 